\title{
Pseudovitelliform Subfoveal Deposit in Waldenström's Macroglobulinemia
}

\author{
Aurélie Brolly $^{\mathrm{a}}$ Claire Janon $^{\mathrm{c}}$ Flavien Precausta $^{\mathrm{c}}$ \\ Jean-Marie Baudet ${ }^{c}$ Salomon Yves Cohen ${ }^{a, b}$ \\ ${ }^{a}$ Department of Ophthalmology, APHP, Hôpital Lariboisière, University Paris \\ Diderot, Sorbonne Paris Cité, ${ }^{b}$ Centre Ophtalmologique d'Imagerie et de Laser, \\ Paris, and ${ }^{\mathrm{C} D e p a r t m e n t}$ of Ophthalmology, Centre Hospitalier Jacques Coeur, \\ Bourges, France
}

\section{Key Words}

Waldenström's macroglobulinemia · Serous retinal detachment · Pseudovitelliform subfoveal deposit - Autofluorescence

\begin{abstract}
Background: Waldenström's macroglobulinemia may be complicated by retinal hemorrhages, retinal vein occlusion, serous macular detachment or macular edema. We report a patient with pseudovitelliform subfoveal deposit complicating Waldenström's macroglobulinemia.
\end{abstract}

Case Report: A 56-year-old man presented with hyperviscosity syndrome due to Waldenström's macroglobulinemia. After systemic therapy, a large serous retinal detachment persisted in the left eye. A pseudovitelliform subfoveal deposit was observed in the right eye.

Conclusion: Pseudovitelliform subfoveal deposits may be part of the spectrum of ocular complications in Waldenström's macroglobulinemia. They could be due to accumulation of macroglobulins.

\section{Introduction}

Waldenström's macroglobulinemia is a non-Hogkin's B cell lymphoplasmocytic lymphoma characterized by bone marrow infiltration and the production of monoclonal IgM. It is complicated by hyperviscosity syndrome due to the accumulation of IgM in the serum. Waldenström's macroglobulinemia may cause clinical cytopenia, hepatosplenomegaly, lymphadenopathy and oronasal bleeding. Its major complications include bone marrow failure, autoimmune cytopenia, large cell lymphoma and 
infections [1]. Retinal symptoms are rare but, when present, due to hyperviscosity. They include retinal hemorrhages and retinal vein occlusion [2]. Serous macular detachment and macular edema have occasionally been reported [3, 4]. To our knowledge, pseudovitelliform subfoveal deposits have not been reported as possible complications of the disease.

\section{Case Report}

A 56-year-old man presented with bilateral decreased vision. His medical history included a carcinoma of the rectum treated with radiotherapy. His best-corrected visual acuity was 20/40 in the right eye and 20/60 in the left eye. Anterior segment examination was normal. Fundus examination revealed bilateral peripheral retinal hemorrhages in the four quadrants, peripheral microaneurisms and venous dilation and tortuosity, suggesting hyperviscosity syndrome. Fluorescein angiography (Spectralis, Heidelberg, Germany) allowed better analysis of the fundus anomalies (fig. 1). Optical coherence tomography (OCT) showed macular edema with retinal serous detachment.

A systemic workup disclosed Waldenström's disease and hyperprotidemia of $138 \mathrm{~g} / \mathrm{l}$ with gammaglobulinemia of $75 \mathrm{~g} / \mathrm{l}$ (monoclonal IgM). Anemia was also present. A bone marrow biopsy confirmed the diagnosis.

Systemic therapy consisted of three sessions of plasmapheresis and three boluses of intravenous steroids. Six sessions of chemotherapy (rituximab and neodex, one session every 4 weeks) were performed. No further treatment was required, but the patient had a 24-month follow-up.

After the systemic therapy, visual acuity improved in the right eye (to 20/25), but not in the left eye $(20 / 60)$, which developed a huge macular serous detachment. As it did not improve after two intravitreal injections of bevacizumab, the patient was referred to us.

Best corrected visual acuity was 20/25 in the right eye and 20/200 in the left eye. The left eye fundus exhibited a large area of serous retinal detachment with accumulation of hyperfluorescent lipofuscin material, as observed by autofluorescence fundus photography, and also loss of central photoreceptors, observed by OCT (Spectralis, Heildelberg, Germany).

Right fundus examination revealed a roundish, subretinal, pseudovitelliform deposit, which displayed uneven hyper-autofluorescence on fundus autofluorescent pictures and uneven hyperfluorescence in fluorescein angiography ( $\underline{\underline{f i g} .2}$ ). There was no change during the 12-month follow-up.

\section{Discussion}

Pseudovitelliform deposits may be observed in various chorioretinal diseases [5] such as adult pseudovitelliform macular dystrophy [6,7], basal laminar drusen [8], reticular pseudodrusen [9], chronic central serous chorioretinopathy, vitreomacular traction and epiretinal membrane [10]. However, to our knowledge, no pseudovitelliform deposits have been reported in cases of Waldenström's macroglobulinemia.

Waldenström's macroglobulinemia is a lymphoproliferative B cell disorder characterized by overproduction of monoclonal IgM. It is known to be one of the causes of hyperviscosity syndrome, which is characterized by retinal hemorrhage, cotton wool spots, peripheral retinal microaneurism, dilated retinal veins, optic disc swelling and retinal venous occlusion. Large and severe chronic retinal detachment, such as that present in the left eye of the patient discussed here, has previously been reported. It is 
considered to result from the presence of proteinaceous osmotic leakage into the subretinal space from the retina and/or the choriocapillaris [11]. Such detachments have also been shown to be resistant to plasmapheresis. Plasmapheresis, however, is very useful in reducing serum hyperviscosity by reducing the concentration of IgM, thus improving venous tortuosity and dilation.

Pseudovitelliform deposits usually consist of debris, including lipofuscin, melanolipofuchsin granules in the RPE and in macrophages, and extracellular material derived from photoreceptor outer segment disks. Such deposits are usually hyperautofluorescent. Depending on their etiology, they are more or less homogenous [5]. In the case discussed, however, the deposit may have been of a different composition and origin, and might have corresponded to an accumulation of subretinal macroglobulins. Indeed, it has been suggested that macroglobulins were responsible for the occurrence of large subretinal detachments observed in Waldenström's macroglobulinemia [11] as it was seen in the left eye of our patient. Therefore, macroglobulins were likely to also be present in the subretinal space of the right eye of our patient. Their amount was probably less important in this eye, explaining the difference of the patterns observed.

\section{Acknowledgement}

This work was supported by CIL-ASSOC, Association for Education and Research, Centre Ophtalmologique d'Imagerie et de Laser, Paris, France.

\section{Disclosure Statement}

The authors declare that there are no conflicts of interest to disclose.
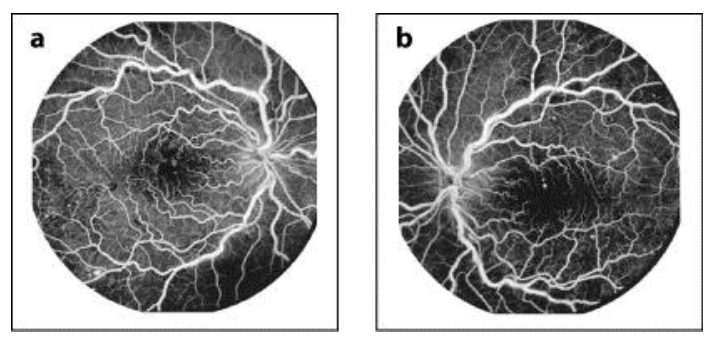

Fig. 1. Fluorescein angiography: Hyperviscosity syndrome characterized by bilateral retinal venous dilation and tortuosity, retinal hemorrhages and peripheral microaneurisms. 

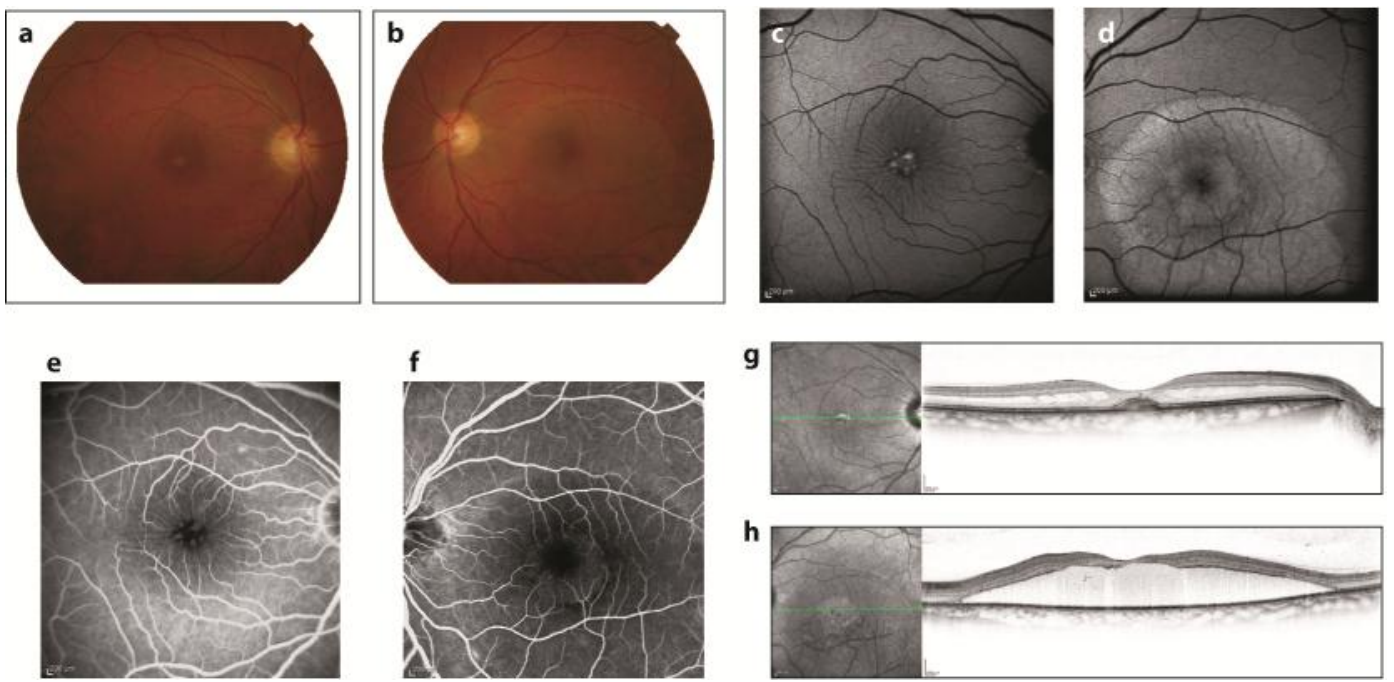

Fig. 2. One year after therapy. The right fundus exhibited a roundish, subretinal, pseudovitelliform deposit (a). The deposit displayed uneven hyper-autofluorescence on a fundus autofluorescent photograph (c) and an uneven staining in fluorescein angiography (e). It was associated with macular edema in an OCT horizontal scan (g). The left eye fundus exhibited a large retinal serous detachment (b), with accumulation of hyper-autofluorescent lipofuscin material in autofluorescence fundus photography (d). Fluorescin angiography was not contributive (f), but OCT showed the absence of central photoreceptors (h).

\section{References}

1 Gertz MA: Waldenström macroglobulinemia: 2011 update on diagnosis, risk stratification, and management. Am J Hematol 2011;86:411-416.

-2 Avashia JH, Fath DF: Bilateral central retinal vein occlusion in Waldenström's macroglobulinemia. J Am Optom Assoc 1989;60:657-658.

-3 Thomas EL, Olk RJ, Markman M, Braine H, Patz A: Irreversible visual loss in Waldenström's macroglobulinaemia. Br J Ophthalmol 1983;67:102-106.

-4 Pilon AF, Rhee PS, Messner LV: Bilateral, persistent serous macular detachments with Waldenström's macroglobulinemia. Optom Vis Sci 2005;82:573-578.

-5 Freund KB, Laud K, Lima LH, Spaide RF, Zweifel S, Yannuzzi LA: Acquired vitelliform lesions: correlation of clinical findings and multiple imaging analyses. Retina 2011;31:13-25.

-6 Puche N, Querques G, Benhamou N, Tick S, Mimoun G, Martinelli D, Soubrane G, Souied EH: Highresolution spectral domain optical coherence tomography features in adult onset foveomacular vitelliform dystrophy. Br J Ophthalmol 2010;94:1190-1196.

-7 Querques G, Forte R, Querques L, Massamba N, Souied EH: Natural course of adult-onset foveomacular vitelliform dystrophy: a spectral-domain optical coherence tomography analysis. Am J Ophthalmol 2011;152:304-313.

-8 Gass JD, Jallow S, Davis B: Adult vitelliform macular detachment occurring in patients with basal laminar drusen. Am J Ophthalmol 1985;99:445-459.

-9 Zweifel SA, Spaide RF, Yannuzzi LA: Acquired vitelliform detachment in patients with subretinal drusenoid deposits (reticular pseudodrusen). Retina 2011;31:229-234.

10 Dupas B, Tadayoni R, Erginay A, Massin P, Gaudric A: Subfoveal deposits secondary to idiopathic epiretinal membranes. Ophthalmology 2009;116:1794-1798.

11 Yannuzzi LA: Waldenström's Macroglobulinemia. The Retinal Atlas. St. Louis, MO, Elsevier-Saunders, 2010, p 476. 